\title{
CONSTRAINTS FROM CHARGE AND COLOUR BREAKING MINIMA IN THE $(\mathrm{M}+1)$ SSM
}

\author{
Ulrich Ellwanger and Cyril Hugonie 1 \\ Laboratoire de Physique Théorique [ \\ Université de Paris XI, Centre d'Orsay, Bâtiment 210, 91405 Orsay Cedex, France
}

\begin{abstract}
We study the constraints on the parameter space of the supersymmetric standard model extended by a gauge singlet, which arise from the absence of global minima of the effective potential with slepton or squark vevs. Particular attention is paid to the so-called "UFB" directions in field space, which are $F$-flat in the MSSM. Although these directions are no longer $F$-flat in the $(\mathrm{M}+1) \mathrm{SSM}$, we show that the corresponding MSSM-like constraints on $m_{0} / M_{1 / 2}$ apply also to the $(\mathrm{M}+1) \mathrm{SSM}$. The net effect of all constraints on the parameter space are more dramatic than in the MSSM. We discuss the phenomenological implications of these constraints.
\end{abstract}

LPTHE Orsay 98-81

January 1999

*email: ellwange@qcd.th.u-psud.fr, cyrilh@qcd.th.u-psud.fr

†Unité mixte de Recherche (UMR 8627) 


\section{Introduction}

In any supersymmetric extension of the standard model the vevs of squarks and charged sleptons have to vanish in order not to break the gauge symmetries $S U(3)_{c}$ and $U(1)_{e m}$ spontaneously. In the last years, many investigations of the MSSM have been carried out in order to find the constraints on the parameter space implied by the absence of such vevs [1] 17]. The aim of the present paper is to find corresponding constraints in the $(\mathrm{M}+1) \mathrm{SSM}$, the supersymmetric extension of the standard model with an additional gauge singlet superfield $S$ in order to replace the $\mu$ term in the superpotential by a vev $\langle S\rangle[18,1,4,10,19]$. (Often the $(\mathrm{M}+1) \mathrm{SSM}$ is also referred to as the NMSSM, the next-to-minimal supersymmetric standard model).

First, we briefly review the dangerous directions in field space and the constraints on the parameter space in the MSSM. Then, in section 2, we compare the most relevant constraints in the MSSM to the ones in the $(\mathrm{M}+1) \mathrm{SSM}$.

Let us, to start with, recall the general structure of the scalar potential $V\left(\varphi_{i}\right)$ of any supersymmetric extension of the standard model: 1) positive semi-definite $F$ and $D$-terms; 2) soft susy breaking mass terms of the form $m_{i}^{2}\left|\varphi_{i}\right|^{2}$ (except for the $B$-term $\mu B H_{1} H_{2}+$ h.c. in the MSSM); 3) soft susy breaking trilinear couplings of the form $\left.A_{i j k} \varphi_{i} \varphi_{j} \varphi_{k} ; 4\right)$ radiative corrections to the effective potential.

These radiative corrections can actually be made to vanish, if an appropriate renormalisation scheme and an appropriate renormalisation scale $Q$ (with $Q \sim\langle\varphi\rangle$ ) for all parameters appearing in $V\left(\varphi_{i}\right)$ are chosen [20]. In practice, where minimal subtraction schemes are employed, the appropriate choice of $Q$ cancels the radiative corrections only for $\langle\varphi\rangle \gg m_{i}, A_{i j k}$, and one is left with the so-called ColemanWeinberg contributions in the regime $\langle\varphi\rangle \sim m_{i}, A_{i j k}$ [20,9,21].

Now, the dangerous directions in field space and the corresponding constraints can be classified as follows:

1) Traditional CCB bounds [1, 4, 9, 10]: here negative contributions to the scalar potential arise from one of the trilinear couplings $A_{i j k} \varphi_{i} \varphi_{j} \varphi_{k}$ (and appropriate phases of the fields $\left.\varphi_{i}\right)$. The $D$-terms can be made to vanish by choosing $\left\langle\varphi_{i}\right\rangle=\left\langle\varphi_{j}\right\rangle=\left\langle\varphi_{k}\right\rangle$, but some of the $F$-terms are always non-zero. Assuming, at the GUT scale, universal trilinear couplings $A_{0}$, scalar masses $m_{0}$ and gaugino masses $M_{1 / 2}$, the absence of 
such minima leads to upper limits on $A_{0}$ as a function of $m_{0}$ and $M_{1 / 2}$, 2, 4, 9, 10,

2) So-called UFB bounds $[8,12-14,16,17]$ : these arise from directions in field space which are both $D$-flat and $F$-flat. Then, the contributions from the trilinear couplings vanish as well. Dangerous directions thus involve fields $\varphi_{i}$, where the soft susy breaking mass $m_{i}^{2}$ is negative (at least at small scales). Since, in order to trigger $S U(2) \times U(1)_{Y}$ symmetry breaking, the mass $m_{1}^{2}$ of the Higgs scalar $H_{1}$ (which couples to the top quark in our convention) is typically negative, one of the fields $\varphi_{i}$ is always given by $H_{1}$. With mass parameters $m_{1}^{2}$ and some $m_{i}^{2}$ taken at the scale $Q=M_{Z}$, one can then find directions $\left\{H_{1}, \varphi_{i}\right\}$ in field space, with respect to which the scalar potential is possibly unstable. If one neglects the scale dependence of $m_{1}^{2}$ and $m_{i}^{2}$, one arrives even at the conclusion that the scalar potential is unbounded from below in these directions (therefore the notion "UFB"). Clearly this conclusion turns out to be erroneous, once the scale dependence of the masses is correctly taken into account, and if the masses squared are assumed to be positive at some large scale $M_{G U T}$.

Nevertheless constraints on the parameters arise from the absence of true minima of the scalar potential in such directions, and these constraints are still called "UFB bounds". Typically, assuming universal soft terms, one obtains lower limits on the ratio $m_{0} / M_{1 / 2}$ of $\mathcal{O}(1)$ (depending to some extent on other parameters like $\tan \beta, h_{t}$ or $M_{1 / 2}$ alone).

3) Improved CCB bounds [6,7, 11, 12]: It has been observed that the directions in field space 1 ) and 2) above (with vanishing $D$-terms) do not necessarily allow to find the absolute minimum of the scalar potential. Allowing for more complicated combinations of vevs $\varphi_{i}$, and some $D$-terms to be non-zero (implying, typically, $\varphi_{i} \neq \varphi_{j}$ ), deeper minima can often be obtained. Sometimes these directions in field space interpolate between the directions 1) and 2) above. Usually, the corresponding constraints depend in a complicated way on many parameters of the MSSM (the soft terms, $\mu$, and the Yukawa couplings) and cannot be represented in the form of universal inequalities among just two or three parameters.

The actual relevance of the bounds 1) - 3) above is not entirely evident: Even if a charge and/or colour breaking minimum of the scalar potential exists, which is deeper than the standard $S U(2) \times U(1)_{Y}$ breaking minimum, this situation can be 
acceptable if the tunneling rate out of the standard minimum is small compared to the age of the universe. Only the cases with large tunneling rates can definitely be excluded. These tunneling rates have been estimated in many papers $[3,5,13,15,16]$ with the result that they are often quite small. Then, the relevance of the bounds 1) - 3) above depends on the early cosmology, i.e. into which minimum we drop after inflation. Since the answer depends on the inflationary potential and the reheating temperature [22], this question cannot be resolved in terms of the parameters of the MSSM above.

In the following we will study the constraints arising from lower lying charge and colour breaking minima of the scalar potential leaving the question of tunneling rates aside. In the next chapter we discuss some particularly relevant directions in field space in some detail, in order to compare the corresponding constraints on the parameters of the MSSM with the $(\mathrm{M}+1) \mathrm{SSM}$.

\section{Constraints in the MSSM and $(\mathrm{M}+1) \mathrm{SSM}$}

First, we consider the most dangerous CCB direction of type 1), which involves the trilinear coupling $h_{e} A_{E} E_{R, 1} L_{1} \cdot H_{2}$. Here $E_{R, 1}$ is the right-handed selectron, $L_{1}$ the left-handed slepton doublet of the first generation, and $H_{2}$ the corresponding Higgs doublet. $h_{e}$ denotes the electron Yukawa coupling with $h_{e} \sim 10^{-5}$. From the absence of a non-trivial minimum of the scalar potential in the $D$-flat direction $\left|E_{R, 1}\right|=\left|L_{1}\right|=\left|H_{2}\right|$ the following inequality among the soft susy breaking terms can be derived in the MSSM [2,4,9, 10]:

$$
A_{E}^{2}<3\left(m_{E}^{2}+m_{L}^{2}+\widehat{m}_{2}^{2}\right)
$$

with $\widehat{m}_{2}^{2}=m_{2}^{2}+\mu^{2}$, and where $m_{E}^{2}, m_{L}^{2}$ and $m_{2}^{2}$ are the soft susy breaking mass terms associated with the three fields above. If the inequality (2.1) is violated, the fields develop vevs of $\mathcal{O}\left(A_{E} / h_{e}\right)$, and the depth of the minimum is of $\mathcal{O}\left(A_{E}^{4} / h_{e}^{2}\right)$. Accordingly the inequality (2.1) has to be imposed at a scale $Q \sim A_{E} / h_{e} \sim 10^{7} \mathrm{GeV}$.

In the $(\mathrm{M}+1) \mathrm{SSM}$, there is no $\mu$ term; an effective $\mu$ term is generated once the vev $\langle S\rangle$ is non-zero. However, once the inequality (2.1) is violated with $\mu=0$, the minimum in the corresponding CCB direction is deeper than the minimum associated 
with a non-zero vev $\langle S\rangle$ (since $h_{e}$ is extremely small). Accordingly the inequality (2.1) holds in the $(\mathrm{M}+1) \mathrm{SSM}$ with $\widehat{m}_{2}^{2}=m_{2}^{2}$. Assuming universal soft terms at the GUT scale, the inequality (2.1) then becomes 10

$$
\left(A_{0}-0.5 M_{1 / 2}\right)^{2}<9 m_{0}^{2}+2.67 M_{1 / 2}^{2} \text {. }
$$

In the MSSM, the inequality (2.2) is weakened by additional positive terms involving $\mu_{0}^{2}$ on the right-hand side.

Also, CCB minima in the stop direction $\left|T_{R}\right|=\left|Q_{3}\right|=\left|H_{1}\right|$ can be considered [2, 1, 9. In the MSSM, the inequality (2.1) has then to be replaced by

$$
A_{t}^{2}<3\left(m_{T_{R}}^{2}+m_{Q_{3}}^{2}+\widehat{m}_{1}^{2}\right)
$$

with $\widehat{m}_{1}^{2}=m_{1}^{2}+\mu^{2}$. If the inequality (2.3) is violated, the fields develop vevs of $\mathcal{O}\left(A_{t} / h_{t}\right) \sim \mathcal{O}\left(M_{Z}\right)$ (accordingly it has to be imposed at the weak scale), and the depth of the minimum is of $\mathcal{O}\left(A_{t}^{4} / h_{t}^{2}\right)$. It is not evident, which of the two inequalities (2.1) and (2.3) is more relevant in the MSSM: the soft masses $m_{T_{R}}^{2}, m_{Q_{3}}^{2}$ and $m_{1}^{2}$ on the right-hand side of $(2.3)$ can be small or even negative at the weak scale; on the other hand, $A_{t}^{2}$ at the weak scale is also usually smaller than $A_{E}^{2}$ at a scale $A_{E} / h_{e}$. Hence a case-by-case analysis is required.

The situation is somewhat simpler in the $(\mathrm{M}+1) \mathrm{SSM}$ : The minimum associated with a non-zero vev $\langle S\rangle$ is always deeper (of $\mathcal{O}\left(A_{k}^{4} / k^{2}\right.$ ), see below) than a minimum in the stop direction. Accordingly, if one compares minima in the stop direction with the standard minimum, a non-zero vev $\langle S\rangle$ has to be taken into account. Thus the inequality (2.3) holds in the $(\mathrm{M}+1) \mathrm{SSM}$ with an effective $\mu$-term included, in contrast to the inequality (2.1). We found that, after imposing (2.2) and the present phenomenological constraints on the parameters on the $(\mathrm{M}+1) \mathrm{SSM}$, the inequality (2.3) with an effective $\mu$-term included is always satisfied automatically.

Improved CCB bounds can be obtained from the absence of vevs in more general directions in field space with non-zero vevs of $T_{R}, Q_{3}, H_{1}, H_{2}$ and sleptons [11,12]. The corresponding constraints cannot be represented in terms of simple inequalities among the bare parameters. However, since $m_{T_{R}}^{2}$ can be negative at the weak scale, nontrivial constraints follow already from the absence of a vev $\left\langle T_{R}\right\rangle$ alone. Approximate analytic expressions for $m_{T_{R}}^{2}$ can be found, e.g., in [10,16, 17,23]. One obtains, from 
these references, $m_{T_{R}}^{2}$ as a function of $M_{1 / 2}, m_{0}, A_{0}$ and $\rho=h_{t}^{2} / h_{t, Q F P}^{2}$ :

$$
m_{T_{R}}^{2} \simeq(1-\rho) m_{0}^{2}-\frac{\rho(1-\rho)}{3}\left(2.24 M_{1 / 2}-A_{0}\right)^{2}+(6.6-2.6 \rho) M_{1 / 2}^{2}
$$

If the right hand side of eq. (2.4) is negative, the depth of the minimum with $\left\langle T_{R}\right\rangle \neq 0$ is $\sim-3 m_{T_{R}}^{4} / 2 g_{3}^{2}$. (We have checked that this minimum is deeper than the one with $\left\langle T_{R}\right\rangle=\left\langle T_{L}\right\rangle \neq 0$, which would cancel the $S U(3) D$-term.) If the soft susy breaking terms and hence $\left|m_{T_{R}}^{2}\right|$ are large, and/or if eq. (2.4) is strongly violated, this stop minimum is deeper than the standard one. We have performed a numerical scan of the complete parameter space of the model, whose details are described in refs. $[10,19]$. For each point in the parameter space, which satisfies the inequality (2.2) and the present phenomenological constraints, we have compared the depth of the potential in the stop direction to the standard minimum (with radiative corrections due to stop/top loops included in both cases). The corresponding constraints on the parameter space are discussed together with our other results below.

Now we turn to the "UFB" directions of type 2), which we will discuss in some detail. Let us recall, to this end, the superpotential of the MSSM:

$$
\begin{aligned}
g_{M S S M}= & \sum_{i} h_{u, i} Q_{i} \cdot H_{1} U_{i R}^{c}+\sum_{i} h_{d, i} Q_{i} \cdot H_{2} D_{i R}^{c} \\
& +\sum_{i} h_{\ell, i} L_{i} \cdot H_{2} E_{i k}^{c}+\mu H_{1} \cdot H_{2}
\end{aligned}
$$

A particularly dangerous $D$ - and $F$-flat direction in field space has been identified by Komatsu [8]. It is associated with vevs of the neutral component of $H_{1}^{0}$, the down squarks of the third generation $D_{3 R}^{c}$ and $D_{3 L}$, and the stau $\widetilde{\nu}_{3}$ :

$$
\begin{gathered}
\left\langle H_{1}^{0}\right\rangle=H_{1}, \\
\left\langle D_{3 R}^{c}\right\rangle=\left\langle D_{3 L}\right\rangle=d \\
\left\langle\widetilde{\nu}_{3}\right\rangle=\widetilde{\nu}
\end{gathered}
$$

For arbitrary $H_{1}, d$ and $\widetilde{\nu}$, the $U(1)_{Y} D$-term and the third component of the $\mathrm{SU}(2)$ $D$-term are, respectively, 


$$
D_{Y}=\frac{g_{1}}{2}\left(d^{2}+H_{1}^{2}-\widetilde{\nu}^{2}\right), \quad D_{S U(2)}^{3}=-\frac{g^{2}}{2}\left(d^{2}+H_{1}^{2}-\widetilde{\nu}^{2}\right) .
$$

From the superpotential (2.5) one finds that the only F-term, which is a priori nonzero, is

$$
F_{H_{2}^{0}}=-h_{d, 3} d^{2}-\mu H_{1}
$$

Hence all $D$ - and $F$-terms vanish for

$$
\begin{aligned}
& H_{1}=-\frac{h_{d, 3}}{\mu} d^{2}, \\
& \widetilde{\nu}^{2}=d^{2}\left(1+\frac{h_{d, 3}^{2}}{\mu^{2}} d^{2}\right) .
\end{aligned}
$$

Then, the only non-vanishing terms in the scalar potential are mass terms, and the potential along this direction becomes

$$
V(d)=\alpha d^{4}+\beta d^{2}
$$

with

$$
\alpha=\frac{h_{d, 3}^{2}}{\mu^{2}}\left(m_{1}^{2}+m_{L_{3}}^{2}\right) \quad, \quad \beta=m_{Q_{3}}^{2}+m_{D_{3}}^{2}+m_{L_{3}}^{2} .
$$

At low scales, $m_{1}^{2}$ is usually negative, and $m_{L_{3}}^{2}$ is the smallest susy breaking mass among the three left-handed slepton doublets. Hence $\alpha$ can well be negative, and $V(d)$ seems to be unbounded from below in this case. However, the appropriate scale dependence of all parameters in $V(d)$ has to be taken into account. Here, this appropriate scale $Q$ is

$$
Q \sim h_{t}\left|H_{1}\right|=h_{t} h_{d, 3} d^{2} / \mu
$$

and $V(d)$ should be written as

$$
V(d)=\alpha\left(Q^{2}(d)\right) d^{4}+\beta\left(Q^{2}(d)\right) d^{2}
$$

Thus, if $\alpha\left(Q^{2}=M_{Z}^{2}\right)$ is negative, but all masses squared and hence $\alpha$ are positive at some large scale, $V(d)$ has a true minimum. 
The same reasoning applies to other $D$ - and $F$-flat directions in field space, as the one considered by Casas et al. in [12], where the down squarks are replaced by sleptons.

Recently, analytic approximations to the potential in these directions have been studied by Abel and Savoy [16], and conditions for non-trivial minima as well as the corresponding tunneling rates have been discussed. Actually it has been found that, even if a deeper minimum in such a "UFB" direction exists, the decay rate of the standard vacuum is usually negligible compared to the age of the universe. Nevertheless, the condition for such a minimum not to be deeper than the standard one implies a lower limit on the ratio $m_{0} / M_{1 / 2}$ of $\mathcal{O}(1)[8,12-14,16,17]$ (assuming, again, universal soft terms); from ref. [16] one finds, as a function of $h_{t}$ or $\tan \beta$,

$$
\frac{m_{0}}{M_{1 / 2}}>0.3-1.0
$$

where the lower bound 0.3 corresponds to larger values of $\tan \beta$. If the inequality (2.14) is violated, one has to assume that the early cosmology places one into the (local and metastable) standard minimum of the potential.

All previous discussions of UFB directions concerned only the MSSM, hence we turn now to the $(\mathrm{M}+1) \mathrm{SSM}$. It involves an additional singlet superfield $S$, and the superpotential reads

$$
\begin{aligned}
g_{(M+1) S S M}= & \sum_{i} h_{u, i} Q_{i} \cdot H_{1} U_{i R}^{c}+\sum_{i} h_{d, i} Q_{i} \cdot H_{2} D_{i R}^{c} \\
& +\sum_{i} h_{\ell, i} L_{i} \cdot H_{2} E_{i k}^{c}+\lambda S H_{1} \cdot H_{2}+\frac{k}{3} S^{3} .
\end{aligned}
$$

Let us have a look at the same direction (2.6) in field space and add, in addition, an arbitrary vev $s$ of the singlet scalar. The $D$-terms are still given by eq. (2.7), but now two $F$-terms are a priori non-zero:

$$
\begin{aligned}
& F_{H_{2}^{0}}=-h_{d, 3} d^{2}-\lambda s H_{1}, \\
& F_{S}=k s^{2} .
\end{aligned}
$$

One easily finds that both $F$-terms vanish only for $s=d=0$. Hence directions of the form (2.6) in field space can no longer be $F$-flat in the $(\mathrm{M}+1) \mathrm{SSM}$, and it seems 
that no "UFB"-bounds exist in this model. We will now show that this conclusion is wrong.

First, we assume that the Yukawa couplings $\lambda$ and $k$ in the superpotential (2.15) are small $\left(\lambda, k \lesssim 10^{-2}\right)$, as it is the case in most of the parameter space of the model 10,18, 19. Assuming, in addition, vevs of $H_{1}$ and $H_{2}$ of $\mathcal{O}\left(M_{Z}\right)$, the vev of $s$ is determined to a high precision by the terms $V_{s}(s)$ in the scalar potential which depend solely on $s$ :

$$
V_{s}(s)=k^{2} s^{4}+\frac{2}{3} k A_{k} s^{3}+m_{s}^{2} s^{2}
$$

The global minimum of $V_{s}$ is assumed at

$$
\bar{s}=-\frac{A_{k}}{4 k}\left(1+\sqrt{1-\frac{8 m_{s}^{2}}{A_{k}^{2}}}\right)
$$

provided the parameters $A_{k}, m_{s}$ satisfy

$$
A_{k}^{2} \geq 9 m_{s}^{2}
$$

If one plugs the vev (2.18) of $s$ back into the complete scalar potential of the $(\mathrm{M}+1) \mathrm{SSM}$, one obtains

$$
V_{(M+1) S S M}\left(\varphi_{i}\right)=\bar{V}_{M S S M}\left(\varphi_{i}\right)+\lambda^{2}\left(H_{1} \cdot H_{2}\right)^{2}+V_{s}(\bar{s})
$$

Here $\bar{V}_{M S S M}$ denotes the scalar potential of the MSSM with effective $\mu$ and $B$ terms given by

$$
\begin{aligned}
& \bar{\mu}=\lambda \bar{s} \\
& \bar{B}=A_{\lambda}+k \bar{s} .
\end{aligned}
$$

Let us now consider the potential in "UFB" directions like (2.6). With $\mathrm{H}_{2}=0$, the potential can be written as

$$
V_{(M+1) S S M}\left(H_{1}, d, \widetilde{\nu}, s\right)=\bar{V}_{M S S M}\left(H_{1}, d, \widetilde{\nu}, s\right)+V_{s}(s)
$$

where $V_{(M+1) S S M}$ depends on $s$ through the effective $\mu$ term given in (2.21). In principle, $V_{(M+1) S S M}$ should be minimized with respect to $H_{1}, d, \widetilde{\nu}$ and $s$, and the depth 
of the corresponding minimum should be compared to the depth of the "physical" minimum with $d=\widetilde{\nu}=0$ (but $H_{1}, H_{2}$ and $s \neq 0$ ). Instead, we will now compare the depths of the effective potential (with radiative corrections included) at three different points in field space:

Point A: the "physical" vacuum with $\langle s\rangle=s_{\text {phys }}$ (which is possibly, but not necessarily close to $\bar{s}$ of eq. (2.18)).

Point B: the minimum of $V_{(M+1) S S M}\left(H_{1}, d, \widetilde{\nu}, s_{\text {phys }}\right)$ with respect to $H_{1}, d$ and $\widetilde{\nu}$, where $s$ is kept fixed at $s_{\text {phys }}$.

Point C: the true minimum of $V_{(M+1) S S M}\left(H_{1}, d, \widetilde{\nu}, s\right)$ with respect to $H_{1}, \mathrm{~d}, \widetilde{\nu}$ and $\mathrm{s}$.

First we compare the depths of the points A and B: If the effective MSSM (with $\left.\bar{\mu}=\lambda s_{\text {phys }}\right)$ has a "UFB" problem, point B is lower than point $\mathrm{A}$. The analysis of the constraints on the parameters, which follow from the condition that point $\mathrm{B}$ is not lower than point A, proceeds as in the MSSM: the fact that $V_{(M+1) S S M}\left(H_{1}, d, \widetilde{\nu}, s_{\text {phys }}\right)$ contains an additional "constant" term $V_{s}\left(s_{\text {phys }}\right)$ plays no role, since this term contributes equally to the CCB minima and the physical minimum, and does hence not affect the comparison of their respective depths.

Second, point $\mathrm{C}$ is, by construction, always deeper than point B. (The true minimum of $V_{(M+1) S S M}\left(H_{1}, d, \widetilde{\nu}, s\right)$ can well be assumed for $s=d=0$.) It is possible, although quite involved, to compare directly the depths of the points A and C. However, and quite trivially, a necessary condition for point $\mathrm{C}$ not to be deeper than point A is that point $\mathrm{B}$ has not to be deeper than point A. The MSSM like constraints, which are required for point $\mathrm{B}$ not to be deeper than point $\mathrm{A}$, are thus necessary for point $\mathrm{C}$ not to be deeper than point $\mathrm{A}$, and for the physical minimum to be the deepest one. Accordingly the MSSM bound (2.14) on the ratio $m_{0} / M_{1 / 2}$ applies also to the $(\mathrm{M}+1) \mathrm{SSM}$ with universal soft terms, if one disregards CCB minima in "UFB" directions which are deeper than the standard minimum (although the standard minimum would be quasi stable).

Possibly stronger constraints on the parameter space of the $(M+1) \mathrm{SSM}$ could be obtained by comparing directly the depths of point $\mathrm{A}$ with point $\mathrm{C}$, but in the following we restrict ourselves to the conclusions which can be drawn from the MSSM like analysis (the comparison of the depths of points A and B).

The constraints (2.14) on the parameter space of the $(M+1)$ SSM (with universal 
soft terms) are more dramatic than in the MSSM: For small Yukawa couplings $\lambda, k$ the soft terms $A_{k}, m_{s}^{2}$ of the $(\mathrm{M}+1) \mathrm{SSM}$ are only weakly renormalized between the scales $M_{G U T}$ and $M_{Z}$, and the necessary inequality (2.19) becomes, in terms of $A_{0}$ and $m_{0}$,

$$
\left|A_{0}\right| \gtrsim 2.9 m_{0}
$$

Clearly, the $(\mathrm{M}+1) \mathrm{SSM}$ inequality (2.23), together with (2.14), turns into a lower bound on the ratio $\left|A_{0}\right| / M_{1 / 2}$ :

$$
\left|A_{0}\right| / M_{1 / 2} \gtrsim 0.9-2.9
$$

On the other hand, the inequality (2.2) provides upper bounds on $\left|A_{0}\right|$ (which are somewhat weaker for $A_{0}$ positive; therefore the constraints discussed below apply actually to $\left.\left|A_{0}\right|\right)$. The remaining region in the parameter space of the $(\mathrm{M}+1) \mathrm{SSM}$ is thus quite constraint, and can be represented in the $A_{0} / m_{0}-m_{0} / M_{1 / 2}$ plane in Fig. 1: Here, the vertical full and dashed lines at $m_{0} / M_{1 / 2}=0.3$ and 1.0 respectively represent the lower limits (2.14) on this ratio, which depend on $\tan \beta$ or $h_{t}$. These bounds apply to the MSSM as well as to the (M+1)SSM. The lower limit (2.23) on $A_{0} / m_{0}$ is represented by a horizontal line, and is specific to the $(\mathrm{M}+1) \mathrm{SSM}$. (For large Yukawa couplings $\lambda, k \gtrsim 10^{-2}$, where the renormalisation of $A_{k}$ and $m_{s}$ can be non-negligible, this lower limit can be somewhat weaker). The upper limit on $A_{0} / m_{0}$ from the inequality (2.2) is represented by a full line. (In the MSSM this upper limit on $A_{0} / m_{0}$ is weaker and depends on $\mu$, cf. the remark below (2.2) ).

Non-vanishing vevs of $\left\langle T_{R}\right\rangle$ are possible (but not necessarily deeper than the standard minimum) as soon as $m_{T_{R}}^{2}$ is negative. Within the remaining regions in the parameter space the value of $\rho$, which minimizes $m_{T_{R}}^{2}$, is $\rho \simeq 0.75$. The dotted line in Fig. 1, which limits $m_{0} / M_{1 / 2}$ from above, corresponds to $m_{T_{R}}^{2}=0$ according to eq. (2.4) with this value of $\rho$ : To the right of this line, $m_{T_{R}}^{2}$ can be negative (if $\rho$ is close to 0.75$)$, and a minimum with $\left\langle T_{R}\right\rangle \neq 0$ can even be deeper than the standard one if, at the same time, the scale of the soft terms and hence $\left|m_{T_{R}}^{2}\right|$ is large compared to $M_{Z}^{2}$

Clearly, the dotted line does not represent a strict upper bound on $m_{0} / M_{1 / 2}$. However, for several reasons the number of allowed points in the parameter space 
decreases as we move to the right of the dotted line (towards larger values of $m_{0} / M_{1 / 2}$ ): First, for $\lambda, \kappa \lesssim 10^{-2}$, as it is the case in most of the parameter space, the lower limit on $A_{0} / m_{0}$ from eq. (2.19) is 3.0 rather than the more generous one of 2.9 indicated in eq. (2.23). Since the upper limit on $A_{0} / m_{0}$ from eq. (2.2) approaches also 3.0 for $m_{0} / M_{1 / 2}$ large, quite some fine-tuning is required in this regime. Second, since here we have $M_{1 / 2}<<m_{0},\left|A_{0}\right|$, it becomes increasingly difficult to satisfy the phenomenological lower limits on the chargino and/or gluino masses. Finally, in order to keep $m_{T_{R}}^{2}$ positive or not too negative, one finds from eq. (2.4) that $\rho$ has to approach 1 for $m_{0}>>M_{1 / 2}$. Then it becomes difficult to satisfy the phenomenological upper bound on the top quark mass. (The corresponding precise constraint on $\rho$ is, however, quite sensitive to 2 loop contributions to the RGEs and the effective potential.)

From our numerical scan of the parameter space of the $(\mathrm{M}+1) \mathrm{SSM}$ employing 1 loop RGEs and the 1 loop Coleman-Weinberg corrections to the effective potential (the unknown threshold corrections at the GUT scale make a consistent 2 loop analysis impossible) we find, due to the combined constraints listed above, no phenomenologically acceptable points in the parameter space with $m_{0} / M_{1 / 2}>7$. This upper limit is indicated by a dash-dotted line in the region of interest. The dashed region is thus the only remaining one in the $(\mathrm{M}+1) \mathrm{SSM}$, if the standard minimum of the scalar potential is required to be the absolute one, regardless of its decay rate.

What would be the phenomenological implications of these constraints on the parameter space of the $(\mathrm{M}+1) \mathrm{SSM}$ ? First, the lower limit (2.24) on $A_{0}$ effects most directly the singlet sector of the model: For a small Yukawa coupling $\lambda$ the singlet neutralino $\widetilde{S}$ (the singlino), and the singlet CP-even and CP-odd scalars are almost pure states. The mass $M_{\tilde{S}}$ of the singlino is approximately given by $2 k \bar{s}$; after replacing $A_{k}$ and $m_{s}^{2}$ in the expression (2.18) for $\bar{s}$ by $A_{0}$ and $m_{0}^{2}$, the singlino mass satisfies

$$
\left|M_{\tilde{S}}\right| \gtrsim \frac{2}{3}\left|A_{0}\right|
$$

From (2.24) one then finds that the singlino can not be lighter than the lightest neutral gaugino (typically the bino with a mass $M_{\widetilde{B}} \sim .41 M_{1 / 2}$ ). Accordingly a "singlino LSP scenario", which leads to unconventional signatures of sparticle production in the $(\mathrm{M}+1) \mathrm{SSM}$ [19], would not be possible. Similarly, the masses of the CP-even and 
CP-odd quasi singlet scalars are bounded from below by $\left|A_{0}\right| / 3$ and $\left|A_{0}\right|$, respectively, and would be quite heavy.

Second, the lower limits (2.23) and (2.24) on $A_{0}$ affect both the renormalisation and mixings of sfermions with large Yukawa couplings, notably the physical stop masses: Whereas the physical masses $m_{s q}$ of the squarks of the first generations generally satisfy

$$
m_{s q} \gtrsim .9 M_{3}
$$

in terms of the gluino mass $M_{3}$, the mass of the lightest stop $m_{s t 1}$ is even bounded from above in terms of $M_{3}$, according to our scan of the parameter space of the $(\mathrm{M}+1) \mathrm{SSM}$ with CCB and UFB constraints:

$$
m_{s t 1} \lesssim 80 \mathrm{GeV}+.8 M_{3}
$$

Altogether, the phenomenology of the $(\mathrm{M}+1) \mathrm{SSM}$ with CCB and UFB constraints will resemble to a large extent the one of the MSSM - with additional, albeit quite heavy, states in the neutralino and Higgs sectors - and with additional constraints on the parameters and sparticle masses.

\section{Summary and Conclusions}

The aim of the present paper is the study of constraints on the parameter space of the $(\mathrm{M}+1) \mathrm{SSM}$ with universal soft terms, which arise from the absence of global minima of the scalar potential with vevs of sleptons or squarks. We have considered the $D$-flat "CCB" and the conventional "UFB" directions in field space, which are comparatively easy to analyse. The consideration of additional "improved" directions could nothing but strengthen the constraints obtained above.

The most important result is the fact that the MSSM "UFB" bounds on the ratio $m_{0} / M_{1 / 2}$ apply also to the $(\mathrm{M}+1) \mathrm{SSM}$. This is highly non-trivial, since the corresponding dangerous directions in field space are no longer $F$-flat in the $(\mathrm{M}+1) \mathrm{SSM}$. Nevertheless, this result follows in a quite straightforward way after the minimization of the $s$ dependent part of the potential with respect to $s$, neglecting interactions with the non-singlet sector of $\mathcal{O}(\lambda)$. (One can check that the relative error of the 
vacuum energy induced by this procedure is of $\mathcal{O}\left(\lambda^{2}\right)$; eventually, improved bounds in the $(\mathrm{M}+1) \mathrm{SSM}$ could be studied in the regime where $\lambda$ is not small.)

The resulting constraints on the parameter space of the $(M+1) S S M$ are more important than in the MSSM: Since here the soft susy breaking terms have to satisfy the additional inequality $A_{0} \gtrsim 2.9 m_{0}$, a lower limit on $m_{0} / M_{1 / 2}$ from "UFB" bounds implies a lower limit on $A_{0} / M_{1 / 2}$, which has no analog in the MSSM. The constraints on the ratios of soft terms have been summarized in Fig. 1, according to which the allowed range of the ratio $A_{0} / m_{0}$ is narrowed by the lower "UFB" bound on $m_{0} / M_{1 / 2}$. The fact that the ratio $A_{0} / m_{0}$ is confined into a quite narrow region around $\sim 3$ for $m_{0} / M_{1 / 2}$ large, has already been realized in previous analyses of the parameter space of the $(\mathrm{M}+1) \mathrm{SSM}$, where the inequality (2.2) has been taken into account [10,19].

Still, one can avoid most of these constraints - as in the MSSM - if one is ready to accept the standard $S U(2) \times U(1)_{Y}$ symmetry breaking vacuum as a local and not global minimum of the scalar potential. Then, instead, constraints on the early

cosmology - the inflationary potential and the corresponding reheating temperature - can be derived from the condition to end up in the local standard vacuum. The tunneling rates associated with the decay of the standard vacuum have not been considered here, but they will again correspond to the ones of the MSSM for $\lambda$ small.

The phenomenological implications of the CCB and UFB constraints in the $(\mathrm{M}+1) \mathrm{SSM}$ are essentially upper bounds on the lightest stop mass as a function of the gluino mass and, most importantly, lower bounds on the quasi singlet states, which rule out a "singlino LSP scenario". Hence, if the singlino LSP scenario could nevertheless be confirmed through its additional cascade decays and - possibly displaced vertices [19], the metastability of our present vacuum would be established.

\section{Acknowledgement}

It is a pleasure to thank Steven Abel for stimulating discussions. 


\section{Figure Caption}

Fig. 1: Constraints on $A_{0} / m_{0}$ (for any sign of $A_{0}$, cf. the discussion below eq. (2.24)) and $m_{0} / M_{1 / 2}$ from the absence of deeper charge and colour breaking minima in the $(\mathrm{M}+1) \mathrm{SSM}$. The lower limits on $m_{0} / M_{1 / 2}$ from (2.14) are represented by vertical full and dashed lines. The upper and lower limits on $A_{0} / m_{0}$ from (2.2) and (2.23), respectively, are indicated by full lines. The upper limit on $m_{0} / M_{1 / 2}$, which follows from $m_{T_{R}}^{2}>0$ with $\rho=0.75$ from eq. (2.4) is shown as a dotted line, and the upper limit on $m_{0} / M_{1 / 2}$, which follows from our numerical results, by a vertical dash-dotted line. The dashed region is the only allowed one in the $(\mathrm{M}+1) \mathrm{SSM}$. 


\section{References}

[1] J. F. Frère, D. R. T. Jones, S. Raby, Nucl. Phys. B222 (1983) 11.

[2] L. Alvarez-Gaumé, J. Polchinski, M. Wise, Nucl. Phys. B221 (1983) 495.

[3] M. Claudson, L. Hall, I. Hinchliffe, Nucl. Phys. B228 (1983) 501.

[4] J.-P. Derendinger, C. A. Savoy, Nucl. Phys. B237 (1984) 307.

[5] U. Ellwanger, Phys. Lett. B141 (1984) 435.

[6] M. Drees, M. Glück, K. Grassie, Phys. Lett. B157 (1985) 164.

[7] J. Gunion, H. Haber, M. Sher, Nucl. Phys. B306 (1988) 1.

[8] H. Komatsu, Phys. Lett. B215 (1988) 323.

[9] G. Gamberini, G. Ridolfi, F. Zwirner, Nucl. Phys. B331 (1990) 331.

[10] U. Ellwanger, M. Rausch de Traubenberg, C. A. Savoy, Phys. Lett. B315 (1993) 331, and Nucl. Phys. B492 (1997) 21.

[11] P. Langacker, N. Polonsky, Phys. Rev. D50 (1994) 2199.

[12] J. A. Casas, A. Lleyda, C. Muñoz, Nucl. Phys. B471 (1996) 3, Phys. Lett. B380 (1996) 59, Phys. Lett. B389 (1996) 305;

H. Baer, M. Brhlik, D. Castano, Phys. Rev. D54 (1996) 6944;

J. A. Casas, hep-ph/9707475;

S. Abel, B. Allanach, Phys. Lett. B431 (1998) 339;

J. A. Casas, A. Ibarra, C. Muñoz, hep-ph/9810266.

[13] A. Riotto, E. Roulet, Phys. Lett. B377 (1996) 60;

I. Dasgupta, R. Rodemacher, P. Suranyi, hep-ph/9804229.

[14] J. A. Casas, S. Dimopoulos, Phys. Lett. B387 (1996) 107.

[15] A. Kusenko, P. Langacker, G. Segre, Phys. Rev. D54 (1996) 5824.

[16] S. Abel, C. A. Savoy, hep-ph/9803218. 
[17] S. Abel, C. A. Savoy, hep-ph/9809498;

S. Abel, T. Falk, hep-ph/9810297.

[18] P. Fayet, Nucl. Phys. B90 (1975) 104;

H. P. Nilles, M. Srednicki, D. Wyler, Phys. Lett. B120 (1983) 346;

J. Ellis, J. F. Gunion, H. E. Haber, L. Roszkowski, F. Zwirner, Phys. Rev. D39 (1989) 844;

L. Durand, J. Lopez, Phys. Lett. B217 (1989) 463;

M. Drees, Int. J. Mod. Phys. A4 (1989) 3635;

T. Elliott, S. F. King, P. L. White, Phys. Rev. D49 (1994) 2435;

S. F. King, P. L. White, Phys. Rev. D52 (1995) 4183.

[19] U. Ellwanger, C. Hugonie, Eur. Phys. J C5 (1998) 723, and hep-ph/9812427.

[20] S. Coleman, E. Weinberg, Phys. Rev. D7 (1973) 1888;

E. Gildener, Phys. Rev. D13 (1976) 1025;

U. Ellwanger, Nucl. Phys. B238 (1984) 665;

M. Sher, Phys. Rep. 179 (1989) 273.

[21] J. A. Casas, J. K. Espinosa, M. Quiros, A. Riotto, Nucl. Phys. B436 (1995) 3.

[22] A. Strumia, Nucl. Phys. B482 (1996) 24;

T. Falk, K. A. Olive, L. Koszkowski, A. Singh, M. Srednicki, Phys. Lett. B396 (1997) 50.

[23] S. Abel, B. Allanach, Phys. Lett. B415 (1997) 371. 


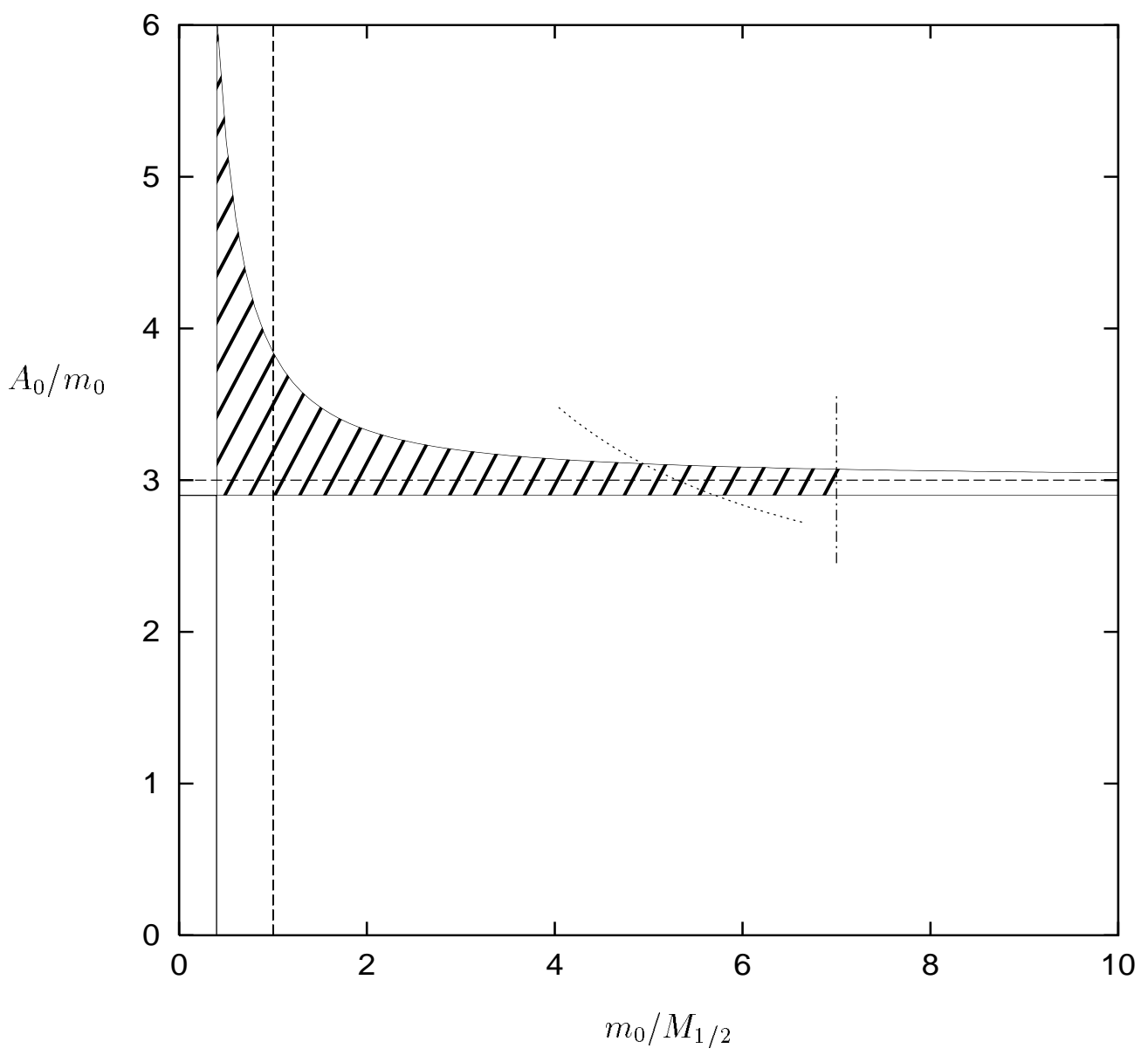

Fig. 1 